\title{
Multichannel Formalism for Positron-Hydrogen Scattering and Annihilation
}

\author{
S L Yakovlev ${ }^{1,2}$, C-Y Hu${ }^{2}$, D. Caballero ${ }^{2}$ \\ ${ }^{1}$ Department of Computational Physics, St Petersburg University, St Petersburg, \\ Russia 198504 \\ E-mail: yakovlev@physics.csulb.edu \\ ${ }^{2}$ Department of Physics and Astronomy, California State University at Long Beach, \\ Long Beach, California 90840
}

\begin{abstract}
A problem to account for the direct electron-positron annihilation in positron-hydrogen scattering above the positronium formation threshold has been resolved within the time independent formalism. The generalization of the optical theorem is derived for the case when an absorption potential is present in the Hamiltonian. With this theorem the annihilation cross section is fully determined by scattering amplitudes. This allows us to separate out analytically the contribution of the positronium formation from the overall annihilation cross section. The rest is determined as the direct annihilation cross section. It is done uniformly below as well as above the positronium formation threshold. The multichannel three-body theory for scattering states in the presence of an imaginary absorption potential is developed in order to compute the direct $e^{+} e^{-}$annihilation amplitude. Special attention has been paid to an accurate definition of the coordinate part of the absorption potential as the properly constructed zero-range potential, which corresponds to the delta-function originated from the first order perturbation theory. The calculated direct annihilation cross section below the positronium formation threshold is in good agreement with results of other authors. The direct annihilation cross section computed with the formalism of the paper shows nonsingular behavior at the positronium formation threshold and is in good agreement with existing data. A number of $e^{+} e^{-}$direct annihilation cross sections and positronium formation cross sections in the energy gap between $\operatorname{Ps}(1 s)$ and $\mathrm{H}(n=2)$ thresholds are reported. A sharp increase in the calculated direct annihilation cross section across the resonant energy is found for all first $\mathrm{S}$ and $\mathrm{P}$-wave Feshbach resonances.

PACS numbers: 36.10.Dr, 34.90.+q
\end{abstract}

Submitted to: J. Phys. B: At. Mol. Opt. Phys. 


\section{Introduction}

In positron-hydrogen collision, if the energy is higher than the positronium formation threshold, we have two genuine different asymptotic channels. The positron may go away leaving the hydrogen atom behind in ground or in an excited state, or the positron and the electron may form a positronium leaving the proton behind. This picture gets modified if we take into account the possibility that the positron and the electron annihilate. In case of rearrangement scattering, when the positron and the electron form positronium in the $\operatorname{Ps}(1 s)$ state, the positron-electron pair annihilates mostly from this state. The lifetime of the $\operatorname{Ps}(1 s)$ state depends on the total spin [1]. In this process, once positronium is formed, the third particle does not affect the annihilation. The direct annihilation occurs without formation of the positronium. In this case the positron interacts with the electron in the field of the proton only when all the three particles are close to each other. Thus, the direct annihilation is a genuine three-body process.

The standard approach treats the $e^{+} e^{-}$annihilation on the basis of the perturbation theory [2, 3]. The annihilation cross section appears as a matrix element of the coalescence operator between scattering states, which are the solution of the unperturbed three-body Coulomb Hamiltonian. At higher energies, this formalism have difficulties. At the positronium formation threshold, the calculated annihilation cross section becomes infinite. There has been a number of attempts to improve theory and to achieve a unified treatment of the positron annihilation and the positronium formation [4, 5, 6]. In one approach, to make the lifetime of the $e^{+} e^{-}$pair finite, an imaginary absorption potential is added to the positron-electron subsystem Hamiltonian [4]. "The loss of particles", due to this potential, is then interpreted as the $e^{+} e^{-}$annihilation. The standard QED formula [2, 3] for the positron annihilation cross section suggests the coordinate part of the absorption potential to be the three-dimensional Dirac delta function depending on the relative position vector between the positron and the electron. Subsequently, this kind of absorption potential was used for computing the annihilation cross section above the positronium formation threshold [ [5, 7, 8]. The three-dimensional delta-function is too singular to be an ordinary potential in the Schrödinger equation [9, 10, 11]. In the actual calculations in Refs. [4, 5, 7, 8] with the delta potential, this singularity was smoothed out. Nevertheless, the mathematically sound formulation of using the three-dimensional zero-range potentials in three-body Coulombic systems is still needed. It is done in this paper to complete the theory.

Direct annihilation cross section above the positronium formation threshold is subject of an extensive study [12]. In Ref. [5, 6] it was claimed that the direct process and the annihilation after the positronium formation are inseparable and therefore the notion of the direct annihilation above the threshold is meaningless. In contrast, the direct cross section was defined and computed successfully below and above the Psformation threshold in Ref. [8]. This calculation showed no sign of the presence of any threshold behavior in the energy dependence of the direct annihilation cross section.

This paper is devoted to continue the study of the direct annihilation within the 
time independent formalism introduced in [13]. In section 2 we define the scattering solution for the $e^{+}-\mathrm{H}$ Schrödinger equation in the presence of an absorption potential. Subsequently, the multichannel formalism, which is needed to determine scattering and absorption amplitudes above the positronium formation threshold, is developed. In section 3 we derive the generalization of the optical theorem in the presence of an absorption potential. This optical theorem is used to separate out the annihilation after the positronium formation cross section from the overall absorption cross section. The remaining part is naturally the direct annihilation cross section. The correct form of the zero-range absorption potential is introduced in section 3. Section 4 contains the results of calculations for the direct annihilation. Section 5 concludes the paper.

Throughout the paper we use bold letters for vectors, e.g. $\mathbf{x}$, and non-bold for their magnitudes, e.g. $x=|\mathbf{x}|$. The unit vector pointing out in the direction of the vector $\mathbf{y}$ is denoted as $\hat{\mathbf{y}}=\mathbf{y} / y$. In the paper we work with wave-functions and their components of the form $\Psi(\mathbf{X}, \mathbf{p})$ where $\mathbf{X}$ stands for the configuration coordinate and $\mathbf{p}$ for the momentum of the asymptotic motion. For the sake of clarity of notations and to avoid overloading of formulae we drop very often the configuration space coordinate from the notations of wave-functions and their components leaving only the momentum explicitly.

\section{Three-body scattering formalism}

In this section we apply the three-body multichannel formalism to $e^{+}-\mathrm{H}$ scattering.

\subsection{Three-body scattering in the presence of absorption potential}

We consider the three-body problem with an additional imaginary potential which acts between the positron and the electron. If the center of mass motion is removed, the Hamiltonian in the Jacobi coordinate system takes the form

$$
\begin{aligned}
& H=H^{C}+\mathrm{i} g W_{2}\left(\mathbf{x}_{2}\right), \\
& H^{C}=H_{0}+V_{1}^{C}\left(\mathbf{x}_{1}\right)+V_{2}^{C}\left(\mathbf{x}_{2}\right)+V_{3}^{C}\left(\mathbf{x}_{3}\right), \\
& H_{0}=-\frac{\hbar^{2}}{2 \mu_{i}} \nabla_{\mathbf{x}_{i}}^{2}-\frac{\hbar^{2}}{2 \nu_{i}} \nabla_{\mathbf{y}_{i}}^{2}, \quad V_{i}^{C}\left(\mathbf{x}_{i}\right)=\frac{e_{j} e_{k}}{x_{i}} .
\end{aligned}
$$

Here we assign numbers 1,2 and 3 to the positron, the proton and the electron, respectively, therefore the electric charges are expressed through the unit charge $e$ as $e_{1}=e_{2}=e, e_{3}=-e$. The Jacobi coordinates are defined in terms of particle radiusvectors $\mathbf{r}_{i}$ by the standard formulae

$$
\mathbf{x}_{i}=\mathbf{r}_{j}-\mathbf{r}_{k}, \quad \mathbf{y}_{i}=\mathbf{r}_{i}-\frac{m_{j} \mathbf{r}_{j}+m_{k} \mathbf{r}_{k}}{m_{j}+m_{k}},
$$

and the reduced masses are given by

$$
\mu_{i}=\frac{m_{j} m_{k}}{m_{j}+m_{k}}, \quad \nu_{i}=\frac{m_{i}\left(m_{j}+m_{k}\right)}{m_{i}+m_{j}+m_{k}} .
$$


The potential $\mathrm{i} g W_{2}\left(\mathbf{x}_{2}\right)$ acting between particles of the pair 2 is defined such that $g$ is real and negative, and $W_{2}\left(\mathbf{x}_{2}\right)$ is real and non negative. In this case $\mathrm{i} g W_{2}$ is a complex absorbing potential. We do not specify the coordinate dependence of $W_{2}$ yet, except of requiring that $W_{2}$ is short-ranged.

The Schrödinger equation for the positron-hydrogen scattering reads

$$
\left(H_{0}+V_{1}^{C}-E\right) \Psi^{+}=-\left(V_{2}^{C}+V_{3}^{C}+\mathrm{i} g W_{2}\right) \Psi^{+} .
$$

The scattering solution is defined at real energy $E$ by the asymptotics as $y_{1} \rightarrow \infty$

$$
\Psi^{+} \propto \phi_{1}\left(\mathbf{x}_{1}\right)\left[e^{\mathrm{i} \mathbf{p}_{1} \cdot \mathbf{y}_{1}}+\frac{e^{\mathrm{i} \sqrt{E-\epsilon_{1}} y_{1}}}{y_{1}} F\left(p_{1} \hat{\mathbf{y}}_{1}, \mathbf{p}_{1}\right)\right] .
$$

Here $\phi_{1}\left(\mathbf{x}_{1}\right)$ is the hydrogen ground state wave function with the energy $\epsilon_{1}$, and the incident momentum of the positron $\mathbf{p}_{1}$ is related to the energy by the condition $E=\hbar^{/} 2 \nu_{1} p_{1}^{2}+\epsilon_{1}$. We note, that due to the asymptotics of the wave-function (77), the term $V_{2}^{C}+V_{3}^{C}$ in the right hand side of Eq. (6) is always confined into the region of the configuration space where the hydrogen wave function $\phi_{1}\left(\mathbf{x}_{1}\right)$ is not negligible. This makes the term $V_{2}^{C}+V_{3}^{C}$ short-range-type and hence the asymptotics (7) in the coordinate $\mathbf{y}_{1}$ is free from the Coulomb contribution. This property holds true for all equations we deal with in the paper.

Conventionally [14], the scattering amplitude $F$ can be represented through the wave function. It is done by rewriting Eq. (6) in the integral form and taking the asymptotics $y_{1} \rightarrow \infty$. By doing so, we get the Lippmann-Schwinger equation (LSE)

$$
\Psi^{+}\left(\mathbf{p}_{1}\right)=\Phi_{1}\left(\mathbf{p}_{1}\right)+G_{1}\left(E^{+}\right)\left(V_{2}^{C}+V_{3}^{C}+\mathrm{i} g W_{2}\right) \Psi^{+}\left(\mathbf{p}_{1}\right),
$$

where $E^{+}=E+\mathrm{i} 0, \Phi_{1}\left(\mathbf{p}_{1}\right)=\phi_{1}\left(\mathbf{x}_{1}\right) e^{\mathrm{i} \mathbf{p}_{1} \cdot \mathbf{y}_{1}}$ is the solution to the channel Schrödinger equation

$$
\left(H_{0}+V_{1}^{C}-E\right) \Phi_{1}\left(\mathbf{p}_{1}\right)=0,
$$

and $G_{1}$ is the channel Green's function $G_{1}(z)=\left(z-H_{0}-V_{1}^{C}\right)^{-1}$. The LSE (8) is the integral equation of the form

$$
\begin{aligned}
& \Psi^{+}\left(\mathbf{X}, \mathbf{p}_{1}\right)=\Phi_{1}\left(\mathbf{X}, \mathbf{p}_{1}\right)+ \\
& \int d \mathbf{X}^{\prime} G_{1}\left(\mathbf{X}, \mathbf{X}^{\prime}, E^{+}\right)\left[V_{2}^{C}\left(\mathbf{x}_{2}^{\prime}\right)+V_{3}^{C}\left(\mathbf{x}_{3}^{\prime}\right)+\mathrm{i} g W_{2}\left(\mathbf{x}_{2}^{\prime}\right)\right] \Psi^{+}\left(\mathbf{X}^{\prime}, \mathbf{p}_{1}\right)
\end{aligned}
$$

where $\mathbf{X}=\left\{\mathbf{x}_{1}, \mathbf{y}_{1}\right\}$ and $\mathbf{x}_{2}^{\prime}, \mathbf{x}_{3}^{\prime}$ are supposed to be represented through $\mathbf{x}_{1}^{\prime}, \mathbf{y}_{1}^{\prime}$ by standard transformations of Jacobi coordinates. The asymptotics of $\Psi^{+}\left(\mathbf{X}, \mathbf{p}_{1}\right)$ as $y_{1} \rightarrow \infty$ can easily be evaluated now from (10) by taking the asymptotics of the Green's function

$$
G_{1}\left(\mathbf{X}, \mathbf{X}^{\prime}, E^{+}\right) \propto \frac{-\nu_{1}}{2 \pi \hbar^{2}} \phi_{1}\left(\mathbf{x}_{1}\right) \frac{e^{\mathrm{i} \sqrt{E-\epsilon_{1}} y_{1}}}{y_{1}} \Phi_{1}{ }^{*}\left(\mathbf{X}^{\prime}, \sqrt{E-\epsilon_{1}} \hat{\mathbf{y}}_{1}\right) .
$$

As the result, we get (7) with the following expression for the amplitude $F$

$$
F\left(\mathbf{p}_{1}^{\prime}, \mathbf{p}_{1}\right)=\frac{-\nu_{1}}{2 \pi \hbar^{2}}\left\langle\Phi_{1}\left(\mathbf{p}_{1}^{\prime}\right)\left|V_{2}^{C}+V_{3}^{C}+\mathrm{i} g W_{2}\right| \Psi^{+}\left(\mathbf{p}_{1}\right)\right\rangle
$$


where the matrix element stands for the integral

$$
\begin{aligned}
& \left\langle\Phi_{1}\left(\mathbf{p}_{1}^{\prime}\right)\left|V_{2}^{C}+V_{3}^{C}+\mathrm{i} g W_{2}\right| \Psi^{+}\left(\mathbf{p}_{1}\right)\right\rangle= \\
& \int d \mathbf{X}^{\prime} \Phi_{1}^{*}\left(\mathbf{X}^{\prime}, \mathbf{p}_{1}^{\prime}\right)\left[V_{2}^{C}\left(\mathbf{x}_{2}^{\prime}\right)+V_{3}^{C}\left(\mathbf{x}_{3}^{\prime}\right)+\mathrm{i} g W_{2}\left(\mathbf{x}_{2}^{\prime}\right)\right] \Psi^{+}\left(\mathbf{X}^{\prime}, \mathbf{p}_{1}\right) .
\end{aligned}
$$

The formula (12) suggests that the scattering amplitude can be split into the sum of two terms

$$
F=F^{0}+\mathrm{i} g F^{1},
$$

where $F^{0}$ is exclusively due to the Coulomb interactions between the positron and the hydrogen and $\mathrm{i} g F^{1}$ is due to the absorption potential only. Let us note, that the immediate identification of these amplitudes with pieces of (12) does not lead to the consistent form of the amplitudes $F^{k}$ since the wave-function $\Psi^{+}$itself may be split into two parts similarly to (14). Hence, the contribution from different kinds of interactions cannot be separated on the basis of equation (12). The appropriate way is to rewrite the LSE (8) in the form of distorted wave representation [14]. To this end, let us recast (8) into

$$
\left[I-G_{1}\left(E^{+}\right)\left(V_{2}^{C}+V_{3}^{C}\right)\right] \Psi^{+}\left(\mathbf{p}_{1}\right)=\Phi_{1}+G_{1}\left(E^{+}\right) \mathrm{i} g W_{2} \Psi^{+}\left(\mathbf{p}_{1}\right) .
$$

If the energy is below the positronium formation threshold, the inversion of the operator from the left hand side can be performed with the help of the formulae

$$
\begin{aligned}
& {\left[I-G_{1}\left(E^{+}\right)\left(V_{2}^{C}+V_{3}^{C}\right)\right]^{-1} \Phi_{1}=\Psi^{0+},} \\
& {\left[I-G_{1}(z)\left(V_{2}^{C}+V_{3}^{C}\right)\right]^{-1} G_{1}(z)=G^{C}(z) .}
\end{aligned}
$$

As the result, the LSE (8) takes the form

$$
\Psi^{+}\left(\mathbf{p}_{1}\right)=\Psi^{0+}\left(\mathbf{p}_{1}\right)+G^{C}\left(E^{+}\right) \mathrm{i} g W_{2} \Psi^{+}\left(\mathbf{p}_{1}\right) .
$$

The asymptotic analysis of equation (18) will give us the representations for amplitudes $F^{0}$ and $F^{1}$.

The inhomogeneous term $\Psi^{0+}\left(\mathbf{p}_{1}\right)$ is the outgoing solution to the $e^{+}-\mathrm{H}$ scattering problem without an absorption potential. Following (16), this function is defined by the solution of the LSE

$$
\Psi^{0+}\left(\mathbf{p}_{1}\right)=\Phi_{1}\left(\mathbf{p}_{1}\right)+G_{1}\left(E^{+}\right)\left(V_{2}^{C}+V_{3}^{C}\right) \Psi^{0+}\left(\mathbf{p}_{1}\right) .
$$

Similarly to (8), the asymptotics of the solution has the form

$$
\Psi^{0+}\left(\mathbf{p}_{1}\right) \propto \phi_{1}\left(\mathbf{x}_{1}\right)\left[e^{\mathrm{i} \mathbf{p}_{1} \cdot \mathbf{y}_{1}}+\frac{e^{\mathrm{i} \sqrt{E-\epsilon_{1}} y_{1}}}{y_{1}} F^{0}\left(p_{1} \hat{\mathbf{y}}_{1}, \mathbf{p}_{1}\right)\right]
$$

with the amplitude given by

$$
F^{0}\left(\mathbf{p}_{1}^{\prime}, \mathbf{p}_{1}\right)=\frac{-\nu_{1}}{2 \pi \hbar^{2}}\left\langle\Phi_{1}\left(\mathbf{p}_{1}^{\prime}\right)\left|V_{2}^{C}+V_{3}^{C}\right| \Psi^{0+}\left(\mathbf{p}_{1}\right)\right\rangle .
$$

The Green's function $G^{C}(z)$ in (18) is defined as $G^{C}(z)=\left(z-H^{C}\right)^{-1}$. Its asymptotics as $y_{1} \rightarrow \infty$ reads

$$
G^{C}\left(\mathbf{X}, \mathbf{X}^{\prime}, E^{+}\right) \propto \frac{-\nu_{1}}{2 \pi \hbar^{2}} \phi_{1}\left(\mathbf{x}_{1}\right) \frac{e^{i \sqrt{E-\epsilon_{1}} y_{1}}}{y_{1}} \Psi^{0-*}\left(\mathbf{X}^{\prime}, \sqrt{E-\epsilon_{1}} \hat{\mathbf{y}}_{1}\right)
$$


Introducing this asymptotics into (18) we get the explicit representation for the amplitude $F^{1}$ from (14)

$$
F^{1}\left(\mathbf{p}_{1}^{\prime}, \mathbf{p}_{1}\right)=\frac{-\nu_{1}}{2 \pi \hbar^{2}}\left\langle\Psi^{0-}\left(\mathbf{p}_{1}^{\prime}\right)\left|W_{2}\right| \Psi^{+}\left(\mathbf{p}_{1}\right)\right\rangle .
$$

In these formulae $\Psi^{0-}\left(\mathbf{p}_{1}\right)$ is the solution of the $e^{+}-\mathrm{H}$ scattering problem with incoming boundary conditions $\Psi^{0-}\left(\mathbf{p}_{1}\right) \propto\left[\Psi^{0+}\left(-\mathbf{p}_{1}\right)\right]^{*}$ when only Coulomb interactions are taken into account in the Hamiltonian. The formulae (21) and (23) provide us with the desired representation for $F$ as the sum of two amplitudes, one of which $\left(F^{0}\right)$ is exclusively due to the Coulomb interactions between the positron and the hydrogen and does not depend on the absorption potential and the other one $\left(F^{1}\right)$ is due to the absorption potential only.

The preceding analysis is not applicable if the energy $E$ is higher than the positronium formation threshold. Indeed, whereas the formulae (6-14) remain valid, the inversion of the operator $I-G_{1}\left(E^{+}\right)\left(V_{2}^{C}+V_{3}^{C}\right)$ in the left hand side of (15) cannot

be performed and, as the consequence, the equations (18, 23) cannot be justified. The formal reason is that the homogeneous equation

$$
\chi=G_{1}\left(E^{+}\right)\left(V_{2}^{C}+V_{3}^{C}\right) \chi
$$

now possesses the nontrivial solution $\chi=\Psi^{02}\left(\mathbf{p}_{2}\right)$ such that

$$
\left(H^{C}-E\right) \Psi^{02}\left(\mathbf{p}_{2}\right)=0
$$

with the asymptotics $\Psi^{02}\left(\mathbf{p}_{2}\right) \propto \phi_{2}\left(\mathbf{x}_{2}\right) e^{\mathrm{i} \mathbf{p}_{2} \cdot \mathbf{y}_{2}}$. The latter describes the scattering of the proton off the positronium ground state $\phi_{2}\left(\mathbf{x}_{2}\right)$ with the binding energy $\epsilon_{2}$.

This problem with LSE is well known in the three-body scattering theory [15, 16, 17] and is the manifestation of the general fact that no single LSE specifies the three-body scattering wave function uniquely, if the rearrangement channel is open. The resolution of the problem has been found by transforming LSE into the matrix equations for the components of the wave function. Proper arranging of the interactions between the equations, which guarantees the uniqueness of the solution, leads to the equations known as the Faddeev three-body equations [16, 17, 18]. We adopt this formalism to our case in the next subsection where it is proven that for the energy above the positronium formation threshold, similar to (14), the amplitude $F$ is given by the formula $F=F_{11}^{0}+\mathrm{i} g F_{11}^{1}$ where the amplitudes $F_{11}^{0}$ and $F_{11}^{1}$ will be defined by formulae (51) and (54).

\subsection{Three-body scattering formalism above the positronium formation threshold}

Let us emphasize that the scattering problem with the Hamiltonian (10) always deals with the single-arrangement channel $e^{+}-\mathrm{H}$ due to the presence of an absorption potential. That means the solution to the Schrödinger equation (66) has the singlearrangement asymptotics (7) irrespective that is the energy below or above the Psformation threshold. However, in order to specify the amplitudes $F^{0}$ and $F^{1}$ one needs 
the solutions to SE with the Hamiltonian $H^{C}$. For the energy above the Ps-formation threshold between $\operatorname{Ps}(n=1)$ and $\mathrm{H}(n=2)$ thresholds the Scrödinger equation

$$
H^{C} \Psi^{0 i}=E \Psi^{0 i}
$$

has two kinds of solutions, which are specified by the asymptotics

$$
\begin{aligned}
& \Psi^{0 i}\left(\mathbf{p}_{i}\right) \propto \phi_{i}\left(\mathbf{x}_{i}\right)\left[e^{\mathrm{i} \mathbf{p}_{i} \cdot \mathbf{y}_{i}}+\frac{e^{\mathrm{i} p_{i} y_{i}}}{y_{i}} f_{i i}\right], \quad y_{i} \rightarrow \infty, \\
& \Psi^{0 i}\left(\mathbf{p}_{i}\right) \propto \phi_{k}\left(\mathbf{x}_{k}\right) \frac{e^{\mathrm{i} p_{k} y_{k}}}{y_{k}} f_{k i}, \quad y_{k} \rightarrow \infty, \quad k \neq i .
\end{aligned}
$$

Here the momenta $\mathbf{p}_{i(k)}$ are related to the energy as $E=\hbar^{2} / 2 \nu_{i(k)} p_{i(k)}^{2}+\epsilon_{i(k)}$ and indices $i(k)$ run over the $\{1,2\}$ set. The formulae (27, 28) reflect the fact that now the asymptotic form of the wave function is different in different asymptotic arrangements. This is exactly that property of the three-body wave-function, which cannot be recovered by any single LSE.

In order to take into account the multichannel character of the scattering problem above the rearrangement threshold we use the formalism of Faddeev equations [18]. Since the original formalism is developed for the short range interaction, at the first stage we reformulate the three-body Hamiltonian in such a way that Coulomb interactions are split into long-range and short-range parts [19]

$$
V_{i}^{C}\left(\mathbf{x}_{i}\right)=V_{i}^{l}\left(\mathbf{x}_{i}, \mathbf{y}_{i}\right)+V_{i}^{s}\left(\mathbf{x}_{i}, \mathbf{y}_{i}\right) .
$$

This splitting is made in the three-body configuration space by a smooth splitting function $\zeta_{i}\left(\mathbf{x}_{i}, \mathbf{y}_{i}\right)$ constructed such that $\zeta_{i}\left(\mathbf{x}_{i}, \mathbf{y}_{i}\right)=1$ if $x_{i} / x_{0}<\left(1+y_{i} / y_{0}\right)^{\nu}$ and $\zeta_{i}\left(\mathbf{x}_{i}, \mathbf{y}_{i}\right)=0$ if $x_{i} / x_{0}>\left(1+y_{i} / y_{0}\right)^{\nu}$ for some $x_{0}>0, y_{0}>0$ and $0<\nu<1 / 2$. With such a $\zeta_{i}$ the short- and long-range parts of the Coulomb potentials are defined as

$$
V_{i}^{s}\left(\mathbf{x}_{i}, \mathbf{y}_{i}\right)=\zeta_{i}\left(\mathbf{x}_{i}, \mathbf{y}_{i}\right) V_{i}^{C}\left(\mathbf{x}_{i}\right) ; \quad V_{i}^{l}=V_{i}^{C}-V_{i}^{s} .
$$

The Hamiltonian (1) is then transformed into

$$
H=H^{l}+V_{1}^{s}+V_{2}^{s}+\mathrm{i} g W_{2} ; \quad H^{l}=T+V_{1}^{l}+V_{2}^{l}+V_{3}^{C} .
$$

After this modification, the components of the wave function $\Psi^{+}$are defined by formulae

$$
\begin{aligned}
& \Psi_{1}^{+}=\left(E^{+}-H^{l}\right)^{-1} V_{1}^{s} \Psi^{+}, \\
& \Psi_{2}^{+}=\left(E^{+}-H^{l}\right)^{-1}\left(V_{2}^{s}+\mathrm{i} g W_{2}\right) \Psi^{+},
\end{aligned}
$$

where $E^{+}=E+\mathrm{i} 0$. Two components are enough in our case. Indeed, the potential $V_{3}^{C}$ between the positron and the proton is repulsive and does not supports bound states. Hence, only two asymptotic arrangements are possible, which are covered by components $\Psi_{1,2}^{+}$.

It is straightforward to see that the sum of the components recovers the wave function

$$
\Psi^{+}=\Psi_{1}^{+}+\Psi_{2}^{+}
$$


and the components obey the set of modified Faddeev equations (MFE)

$$
\begin{array}{ll}
\left(E-H^{l}-V_{1}^{s}\right) \Psi_{1}^{+} & =V_{1}^{s} \Psi_{2}^{+}, \\
\left(E-H^{l}-V_{2}^{s}-\mathrm{i} g W_{2}\right) \Psi_{2}^{+} & =\left(V_{2}^{s}+\mathrm{i} g W_{2}\right) \Psi_{1}^{+} .
\end{array}
$$

The important feature of equations (32, 33), with regard to the description of the annihilation, is the fact that now the two-body absorption potential $\mathrm{i} g W_{2}$ is incorporated into equations in two manners. It appears in the diagonal part on the left hand side of the equation (33) and in the coupling term on the right hand side. The diagonal terms of the equations (32, 33) are responsible for the asymptotic configurations in channels 1 and 2, so the absorption potential in the left hand side of (33) is natural to associate with the annihilation after positronium formation. The coupling term in (32, 33) couples equations in the reaction volume, therefore the absorption potential in the right hand side of (33) is the source for the direct annihilation. These associations will be put on the solid ground in the next section.

As in the case of equation (6), the scattering solution to equations (32, 33) is defined by the asymptotics as $y_{1} \rightarrow \infty$

$$
\begin{aligned}
& \Psi_{1}^{+}\left(\mathbf{p}_{1}\right) \propto \phi_{1}\left(\mathbf{x}_{1}\right)\left[e^{\mathrm{i} \mathbf{p}_{1} \cdot \mathbf{y}_{1}}+\frac{e^{\mathrm{i} p_{1} y_{1}}}{y_{1}} F_{11}\right], \\
& \Psi_{2}^{+}\left(\mathbf{p}_{1}\right) \propto 0 .
\end{aligned}
$$

Certainly, for the amplitudes $F_{11}$ and $F$ from (12) the equality holds true

$$
F_{11}=F
$$

as it should be in view of (31). Again, as in the case of equation (6), the solution required by (34, 35) is given by the integral form of MFE (32, 33) (IMFE)

$$
\begin{array}{ll}
\Psi_{1}^{+}\left(\mathbf{p}_{1}\right)=\Phi_{1}^{0+}\left(\mathbf{p}_{1}\right)+ & \left(E^{+}-H^{l}-V_{1}^{s}\right)^{-1} V_{1}^{s} \Psi_{2}^{+}\left(\mathbf{p}_{1}\right), \\
\Psi_{2}^{+}\left(\mathbf{p}_{1}\right)= & \left(E^{+}-H^{l}-V_{2}^{s}-\mathrm{i} g W_{2}\right)^{-1}\left(V_{2}^{s}+\mathrm{i} g W_{2}\right) \Psi_{1}^{+}\left(\mathbf{p}_{1}\right) .
\end{array}
$$

Here $\Phi_{1}^{0+}$ stands for the solution to the channel Schrödinger equation $\left(H^{l}+V_{1}^{s}-E\right) \Phi_{1}^{0+}=$ 0 , which explicit form is

$$
\left(H_{0}+V_{1}-E\right) \Phi_{1}^{0+}=-\left(V_{2}^{l}+V_{3}^{C}\right) \Phi_{1}^{0+} .
$$

Repeating reasoning of (8-13) we get the asymptotics as $y_{1} \rightarrow \infty$

$$
\Phi_{1}^{0+}\left(\mathbf{p}_{1}\right) \propto \Phi_{1}\left(\mathbf{p}_{1}\right)+\phi_{1}\left(\mathbf{x}_{1}\right) \frac{e^{\mathrm{i} p_{1} y_{1}}}{y_{1}} f_{11}^{0}
$$

with

$$
f_{11}^{0}\left(\mathbf{p}_{1}^{\prime}, \mathbf{p}_{1}\right)=\frac{-\nu_{1}}{2 \pi \hbar^{2}}\left\langle\Phi_{1}\left(\mathbf{p}_{1}^{\prime}\right)\left|V_{2}^{l}+V_{3}^{C}\right| \Phi_{1}^{0}\left(\mathbf{p}_{1}\right)\right\rangle .
$$

The IMFE (36, 37) are proven to have the unique solution below as well as above the rearrangement threshold [18, 19]. We use this property to remedy the shortcoming of the LSE approach outlined above in specifying the structure of the amplitude $F$ above the Ps-formation threshold. 
In order to proceed, it is convenient to introduce matrix notations

$$
\begin{aligned}
& \mathbf{H}(\mathrm{i} g)=\left[\begin{array}{cc}
H^{l}+V_{1}^{s} & 0 \\
0 & H^{l}+V_{2}^{s}+\mathrm{i} g W_{2}
\end{array}\right], \\
& \mathbf{V}=\left[\begin{array}{cc}
0 & V_{1}^{s} \\
V_{2}^{s} & 0
\end{array}\right], \quad \mathbf{W}=\left[\begin{array}{cc}
0 & 0 \\
W_{2} & 0
\end{array}\right], \quad \mathbf{I}=\left[\begin{array}{ll}
1 & 0 \\
0 & 1
\end{array}\right] .
\end{aligned}
$$

Thus, the MFE set takes the form

$$
[\mathbf{H}(\mathrm{i} g)-E \mathbf{I}] \boldsymbol{\Psi}\left(\mathbf{p}_{1}\right)=-[\mathbf{V}+\mathrm{i} g \mathbf{W}] \boldsymbol{\Psi}\left(\mathbf{p}_{1}\right),
$$

where the vector solution is constructed from wave function components as $\boldsymbol{\Psi}=$ $\left(\Psi_{1}^{+}, \Psi_{2}^{+}\right)$. The IMFE set (36, 37) in matrix notations reads

$$
\boldsymbol{\Psi}\left(\mathbf{p}_{1}\right)=\boldsymbol{\Phi}^{0+}\left(\mathbf{p}_{1}\right)+\left[E^{+} \mathbf{I}-\mathbf{H}(\mathrm{i} g)\right]^{-1}[\mathbf{V}+\mathrm{i} g \mathbf{W}] \mathbf{\Psi}\left(\mathbf{p}_{1}\right) .
$$

The vector of the inhomogeneous term is defined as $\boldsymbol{\Phi}^{0+}\left(\mathbf{p}_{1}\right)=\left(\Phi_{1}^{0+}, 0\right)$. This equation can be reformulated in the form of distorted wave representation by similar way we made for LSE (8). The equation (42) can be recast into

$$
\left\{\mathbf{I}-\left[E^{+} \mathbf{I}-\mathbf{H}(\mathrm{i} g)\right]^{-1} \mathbf{V}\right\} \boldsymbol{\Psi}\left(\mathbf{p}_{1}\right)=\boldsymbol{\Phi}^{0+}\left(\mathbf{p}_{1}\right)+\left[E^{+} \mathbf{I}-\mathbf{H}(\mathrm{i} g)\right]^{-1} \mathrm{i} g \mathbf{W} \mathbf{\Psi}\left(\mathbf{p}_{1}\right) .
$$

Then, by the use of the formulae

$$
\begin{aligned}
& \left\{\mathbf{I}-\left[E^{+} \mathbf{I}-\mathbf{H}(\mathrm{i} g)\right]^{-1} \mathbf{V}\right\}^{-1} \boldsymbol{\Phi}^{0+}\left(\mathbf{p}_{1}\right)=\mathbf{\Psi}^{0+}\left(\mathbf{p}_{1}\right), \\
& \left\{\mathbf{I}-[z \mathbf{I}-\mathbf{H}(\mathrm{i} g)]^{-1} \mathbf{V}\right\}^{-1}[z \mathbf{I}-\mathbf{H}(\mathrm{i} g)]^{-1}=[z \mathbf{I}-\mathbf{H}(\mathrm{i} g)-\mathbf{V}]^{-1}
\end{aligned}
$$

we arrive at the desired distorted wave representation of the IMFE

$$
\boldsymbol{\Psi}\left(\mathbf{p}_{1}\right)=\Psi^{0+}\left(\mathbf{p}_{1}\right)+\left[E^{+} \mathbf{I}-\mathbf{H}(\mathrm{i} g)-\mathbf{V}\right]^{-1} \mathrm{i} g \mathbf{W} \boldsymbol{\Psi}\left(\mathbf{p}_{1}\right) .
$$

From this equation it is seen that

$$
\boldsymbol{\Psi}\left(\mathbf{p}_{1}\right)=\Psi^{0+}\left(\mathbf{p}_{1}\right)+\Psi^{1+}\left(\mathbf{p}_{1}\right)
$$

where $\boldsymbol{\Psi}^{1+}\left(\mathbf{p}_{1}\right)$ stands for the second term in equation (45). The equation (45) is the direct analog of (18) but, in contrast to that, is well defined below as well as above the Ps-formation threshold and therefore can be used to get the necessary representation for the amplitude $F_{11}$.

The asymptotics of $\boldsymbol{\Psi}\left(\mathbf{p}_{1}\right)$ is formed from contributions of both terms in (46). Let us consider the inhomogeneous term $\Psi^{0+}\left(\mathbf{p}_{1}\right)$. The equation for this term reads

$$
\boldsymbol{\Psi}^{0+}\left(\mathbf{p}_{1}\right)=\boldsymbol{\Phi}^{0+}\left(\mathbf{p}_{1}\right)+\left[E^{+} \mathbf{I}-\mathbf{H}(\mathrm{i} g)\right]^{-1} \mathbf{V} \boldsymbol{\Psi}^{0+}\left(\mathbf{p}_{1}\right)
$$

or

$$
[\mathbf{H}(\mathrm{i} g)+\mathbf{V}] \boldsymbol{\Psi}^{0+}\left(\mathbf{p}_{1}\right)=E \mathbf{I} \Psi^{0+}\left(\mathbf{p}_{1}\right) .
$$

These equations are quite similar to (32, 33) and (36, 37) except the coupling term $\mathbf{V}$, which does not contain the absorption potential. The asymptotics of the solution to 
(48) as $y_{1} \rightarrow \infty$, which follows from (47), is similar to (34, 35) and for the components of $\Psi^{0+}=\left(\Psi_{1}^{0+}, \Psi_{2}^{0+}\right)$ has the form

$$
\begin{aligned}
& \Psi_{1}^{0+}\left(\mathbf{p}_{1}\right) \propto \phi_{1}\left(\mathbf{x}_{1}\right)\left[e^{\mathrm{i} \mathbf{p}_{1} \cdot \mathbf{y}_{1}}+\frac{e^{\mathrm{i} p_{1} y_{1}}}{y_{1}} F_{11}^{0}\right] \\
& \Psi_{2}^{0+}\left(\mathbf{p}_{1}\right) \propto 0 .
\end{aligned}
$$

As it was demonstrated above, the formula for the amplitude $F_{11}^{0}$ should be derived by taking asymptotics of the right hand side of (47) as $y_{1} \rightarrow \infty$. A minor difference from what we have demonstrated above is that the nontrivial contribution into the amplitude comes from not only the Green's function source term but also from the driving term due to (39). So that, the amplitude $F_{11}^{0}$ is given by

$$
F_{11}^{0}\left(\mathbf{p}_{1}^{\prime}, \mathbf{p}_{1}\right)=\frac{-\nu_{1}}{2 \pi \hbar^{2}}\left[\left\langle\Phi_{1}\left(\mathbf{p}_{1}^{\prime}\right)\left|V_{2}^{l}+V_{3}^{C}\right| \Phi_{1}^{0+}\left(\mathbf{p}_{1}\right)\right\rangle+\left\langle\Phi_{1}^{0+}\left(\mathbf{p}_{1}^{\prime}\right)\left|V_{1}^{s}\right| \Psi_{2}^{0+}\left(\mathbf{p}_{1}\right)\right\rangle\right] .(51
$$

Now we consider the $\Psi^{1+}\left(\mathbf{p}_{1}\right)$ term. Its asymptotics as $y_{1} \rightarrow \infty$ is defined by the Green's function $\mathbf{G}(z)=[z \mathbf{I}-\mathbf{H}(\mathrm{i} g)-\mathbf{V}]^{-1}$. This function is a genuine three-body quantity and the asymptotics of its matrix elements as $y_{i} \rightarrow \infty$ may be written in the form

$$
G_{i j}\left(\mathbf{X}, \mathbf{X}^{\prime}, E^{+}\right) \propto \frac{-\nu_{i}}{2 \pi \hbar} \phi_{i}\left(\mathbf{x}_{i}\right) \frac{e^{\mathrm{i} \sqrt{E-\epsilon_{i}} y_{i}}}{y_{i}} \Upsilon_{j}^{0-*}\left(\mathbf{X}^{\prime}, \sqrt{E-\epsilon_{i}} \hat{\mathbf{y}}_{i}\right),
$$

where $\Upsilon_{j}^{0-}$ is the eigenfunction of the adjoint to the operator from the left hand side of (48). This asymptotics has different character for $i=1$ and $i=2$. In the first case $G_{1 j}$ does not vanish with $y_{1}$ large since $E-\epsilon_{1}$ is real nonnegative. If $i=2$, the Positronium binding energy $\epsilon_{2}$ becomes complex when the absorption potential is introduced into the $e^{+} e^{-}$Hamiltonian. That makes the relative momentum $p_{2}=\sqrt{E-\epsilon_{2}}$ complex, i.e. $p_{2}=p_{2}^{r}+\mathrm{i} p_{2}^{i}$. So, the asymptotics of $G_{2 j}$ vanish exponentially. Thus, only $\Psi_{1}^{1+}$ component has the nontrivial asymptotics

$$
\Psi_{1}^{1+}\left(\mathbf{p}_{1}\right) \propto \phi_{1}\left(\mathbf{x}_{1}\right) \frac{e^{\mathrm{i} p_{1} y_{1}}}{y_{1}} \mathrm{i} g F_{11}^{1}
$$

with the amplitude $F_{11}$ given by

$$
F_{11}^{1}\left(\mathbf{p}_{1}^{\prime}, \mathbf{p}_{1}\right)=\frac{-\nu_{1}}{2 \pi \hbar^{2}}\left\langle\Upsilon_{2}^{0-}\left(\mathbf{p}_{1}^{\prime}\right)\left|W_{2}\right| \Psi_{1}^{+}\left(\mathbf{p}_{1}\right)\right\rangle .
$$

Here $\Upsilon_{2}^{0-}$ is the second component of the solution to the adjoint equation to (48)

$$
\left[\mathbf{H}(-\mathbf{i} g)+\mathbf{V}^{T}-E \mathbf{I}\right] \boldsymbol{\Upsilon}^{0-}\left(\mathbf{p}_{1}\right)=0
$$

being defined by the integral form

$$
\mathbf{\Upsilon}^{0-}\left(\mathbf{p}_{1}\right)=\boldsymbol{\Phi}^{0-}\left(\mathbf{p}_{1}\right)+\left[E^{-} \mathbf{I}-\mathbf{H}(-\mathrm{i} g)\right]^{-1} \mathbf{V}^{T} \mathbf{\Upsilon}^{0-}\left(\mathbf{p}_{1}\right)
$$

In this equation $E^{-}=E-\mathrm{i} 0, \mathbf{V}^{T}$ means transposed matrix and $\boldsymbol{\Phi}^{0-}=\left(\Phi_{1}^{0-}, 0\right)$ where $\Phi_{1}^{0-}$ is the solution to (38) with incoming boundary conditions. The solution to (55) in the case of $g=0$ takes the very simple form, i.e. $\Upsilon_{1}^{0}=\Upsilon_{2}^{0}=\Psi^{0}$ where $\Psi^{0}$ is the three-body wave-function for the pure Coulomb problem. So, $\Psi^{0}$ obeys the Schrödinger equation (26) and can be constructed from wave-function Faddeev components (29, 30) at $g=0$ as $\Psi^{0}=\Psi_{1}^{00}+\Psi_{2}^{00}$. The detailed information on this and other features of the 
matrix equations as MFE and IMFE and matrix Green's functions, which is necessary for evaluations made above, can be found in Ref. [20].

The formulae (51, 54) determine the ingredients of the amplitude $F_{11}$ uniformly above as well as below the rearrangement threshold of the positronium formation. It is possible to show by preforming backwards transformations that below the Ps-formation threshold, where the representations (14, 21, 23) are valid, the equality

$$
F_{11}^{0}+\mathrm{i} g F_{11}^{1}=F^{0}+\mathrm{i} g F^{1}=F
$$

holds true. It is important to note, that from the analysis of the equation (47) it follows

$$
F_{11}^{0}=F^{0}+\mathcal{O}(g) .
$$

This means that the respective terms in the left and right hand sides of the first equation in the chain (57) are not identical. In fact the amplitude $F_{11}^{0}$ takes into account not only the Coulomb interaction between the positron and the hydrogen, as $F^{0}$ does, but also the possibility of $e^{+} e^{-}$annihilation after the positronium formation. That is due to the presence of the absorption potential in the diagonal part of the equations (48). This means that below the positronium formation threshold the annihilation after virtual formation of the positronium is incorporated into the $F_{11}^{0}$ amplitude and consequently $F_{11}^{1}$ is the pure direct annihilation amplitude. It is worth mentioning again, that the formulae (51, 54) determine the amplitudes uniformly below as well as above the positronium formation threshold, whereas it is not true for representations (21, 23). They are valid only below the positronium formation threshold.

\section{Optical theorem in the presence of absorption and annihilation cross section}

\subsection{Optical theorem}

The standard optical theorem for Hermitian Hamiltonians is nothing but the manifestation of the flux conservation, what is equivalent to the unitarity of the Smatrix. The absorption potential breaks the Hermiticity and the scattering is not unitary. The lack of unitarity is the measure of how much of the flux is absorbed and in the case of the annihilation is the way to determine the annihilation cross section. There is extensive literature on the optical theorem but [14] is the most suitable for our purpose. Following this approach by multiplying the SE (6) by complex conjugate wave function $\Psi^{+*}$ and subtracting the complex conjugated SE multiplied by $\Psi^{+}$we arrive at the equality

$$
\Psi^{+*} H_{0} \Psi^{+}-\Psi^{+} H_{0} \Psi^{+*}=-2 \mathrm{i} g W_{2}\left|\Psi^{+}\right|^{2} .
$$

Integrating over the domain $\Omega_{R}=\left\{y_{1} \leq R\right\}$, using the Green's formula and taking the limit as $R \rightarrow \infty$, we get the following result

$$
-2 \mathrm{i} g \int d \mathbf{x}_{1} d \mathbf{y}_{1} W_{2}\left|\Psi^{+}\right|^{2}=\frac{\hbar^{2}}{2 \nu_{1}} \lim _{R \rightarrow \infty} \int R^{2} d \hat{\mathbf{y}}_{1} \int d \mathbf{x}_{1}\left\{\Psi^{+}, \Psi^{+^{*}}\right\},
$$


which represents the balance of the flux. Here the Wronskian $\left\{\Psi^{+}, \Psi^{+*}\right\}=\Psi^{+} \partial_{y_{1}} \Psi^{+*}-$ $\Psi^{+*} \partial_{y_{1}} \Psi^{+}$has to be taken at the condition $y_{1}=R$. Then, using the asymptotic form of $\Psi^{+}$given in (7), the normalization of the ground-state wave functions $\phi_{1}\left(\mathbf{x}_{1}\right)$ and the weak asymptotics of the plain wave (see for example [21])

$$
e^{\mathrm{i} \mathbf{p} \cdot \mathbf{y}} \propto \frac{2 \pi}{\mathrm{i} p y}\left[-\delta(\hat{\mathbf{p}}+\hat{\mathbf{y}}) e^{-\mathrm{i} p y}+\delta(\hat{\mathbf{p}}-\hat{\mathbf{y}}) e^{\mathrm{i} p y}\right]
$$

we finally arrive at the optical theorem in the presence of absorption

$$
\frac{2 \nu_{1}(-g)}{\hbar^{2} p_{1}} \int d \mathbf{x}_{1} d \mathbf{y}_{1} W_{2}\left|\Psi^{+}\right|^{2}=\frac{4 \pi}{p_{1}} \Im m F\left(\mathbf{p}_{1}, \mathbf{p}_{1}\right)-\int d \hat{\mathbf{y}}\left|F\left(p_{1} \hat{\mathbf{y}}, \mathbf{p}_{1}\right)\right|^{2} .
$$

The positive quantity in the left hand side of (61) is the absorption cross section which determines in our case the overall $e^{+} e^{-}$annihilation cross section due to the direct process as well as due to the annihilation after (virtual if $E<\epsilon_{2}$ or actual if $E>\epsilon_{2}$ ) positronium formation

$$
\sigma^{a}=\frac{2 \nu_{1}(-g)}{\hbar^{2} p_{1}} \int d \mathbf{x}_{1} d \mathbf{y}_{1} W_{2}\left|\Psi^{+}\right|^{2}
$$

So that, the overall annihilation cross section can be computed either by the integral (62) or by the expression in the right hand side of (61), if the total amplitude $F$ is in possession.

In order to go beyond the standard formulation (61, 62) one needs to use the detailed structure of the amplitude $F$. In our case it is the representation (57)

$$
F=F_{11}^{0}+\mathrm{i} g F_{11}^{1}
$$

which leads to the following form of (61)

$$
\begin{aligned}
& \sigma^{a}=\sigma_{2}^{a}+\sigma_{1}^{a} \\
& \sigma_{2}^{a}=\frac{4 \pi}{p_{1}} \Im \mathrm{m} F_{11}^{0}\left(\mathbf{p}_{1}, \mathbf{p}_{1}\right)-\int d \hat{\mathbf{y}}\left|F_{11}^{0}\left(p_{1} \hat{\mathbf{y}}, \mathbf{p}_{1}\right)\right|^{2} \\
& \sigma_{1}^{a}=\frac{4 \pi}{p_{1}}(-g) \Re \mathrm{e} F_{11}^{1}\left(\mathbf{p}_{1}, \mathbf{p}_{1}\right)- \\
& 2(-g) \int d \hat{\mathbf{y}} \Im \mathrm{m} F_{11}^{0}\left(p_{1} \hat{\mathbf{y}}, \mathbf{p}_{1}\right) F_{11}^{1 *}\left(p_{1} \hat{\mathbf{y}}, \mathbf{p}_{1}\right)-g^{2} \int d \hat{\mathbf{y}}\left|F_{11}^{1}\left(p_{1} \hat{\mathbf{y}}, \mathbf{p}_{1}\right)\right|^{2} .
\end{aligned}
$$

The quantities $\sigma_{1}^{a}$ and $\sigma_{2}^{a}$ have meaning of annihilation cross sections for the direct process and the process of the annihilation after the positronium formation. To make this statement sound, let us show that $\sigma_{2}^{a}$ is the cross section of the after the positronium formation annihilation. Thus, the remaining part of $\sigma^{a}$, what is $\sigma_{1}^{a}$, should be interpreted as the direct annihilation cross section.

It is apparent, that the equation (64) is the optical theorem formulated for the equation (48). Indeed, multiplying the equation (48) by $\boldsymbol{\Upsilon}^{+0}$ from the left and subtracting the equation for $\Upsilon^{+0}$

$$
\left[\mathbf{H}(\mathrm{i} g)+\mathbf{V}^{T}-E \mathbf{I}\right] \mathbf{\Upsilon}^{0+}\left(\mathbf{p}_{1}\right)=0
$$

multiplied by $\Psi^{0+}$ from the right and making obvious cancelations we get

$$
\left\langle\mathbf{\Upsilon}^{0+}, H_{0} \mathbf{I} \Psi^{0+}\right\rangle-\left\langle H_{0} \mathbf{I} \boldsymbol{\Upsilon}^{0+}, \Psi^{0+}\right\rangle=2 \mathrm{i} g\left\langle\mathbf{\Upsilon}^{0+}, \mathbf{D} \boldsymbol{\Psi}^{0+}\right\rangle
$$


Here $\mathbf{D}$ is a diagonal matrix $\operatorname{diag}\left\{0, W_{2}\right\}$ and $\langle.,$.$\rangle is the scalar product in the two$ dimensional complex space $\mathbf{C}^{2}$ of wave-function components. Repeating argumentations which led us to the formula (61), we arrive at the equality

$$
\begin{aligned}
& \frac{2 \nu_{1}(-g)}{\hbar^{2} p_{1}} \int d \mathbf{x}_{1} d \mathbf{y}_{1} \Upsilon_{2}^{0+*}\left(\mathbf{p}_{1}\right) W_{2} \Psi_{2}^{0+}\left(\mathbf{p}_{1}\right)= \\
& \frac{4 \pi}{p_{1}} \Im \mathrm{m} F_{11}^{0}\left(\mathbf{p}_{1}, \mathbf{p}_{1}\right)-\int d \hat{\mathbf{y}}\left|F_{11}^{0}\left(p_{1} \hat{\mathbf{y}}, \mathbf{p}_{1}\right)\right|^{2} .
\end{aligned}
$$

This is the optical theorem for equation (48) and the annihilation cross section $\sigma_{2}^{a}$ can be expressed now in terms of the left hand side as

$$
\sigma_{2}^{a}=\frac{2 \nu_{1}(-g)}{\hbar^{2} p_{1}} \int d \mathbf{x}_{1} d \mathbf{y}_{1} \Upsilon_{2}^{0+*}\left(\mathbf{p}_{1}\right) W_{2} \Psi_{2}^{0+}\left(\mathbf{p}_{1}\right) .
$$

To elucidate the further meaning of the cross section $\sigma_{2}^{a}$ it is instructive to consider the limiting case as $g \rightarrow 0$. In the limit no absorption potential is present, scattering becomes unitary and amplitude $F_{11}^{0}$ coincides with $f_{11}$ from (27). The standard unitary variant of the optical theorem for the amplitude $f_{11}$ has the form

$$
\frac{4 \pi}{p_{1}} \Im \mathrm{m} f_{11}\left(\mathbf{p}_{1}, \mathbf{p}_{1}\right)-\int d \hat{\mathbf{y}}\left|f_{11}\left(p_{1} \hat{\mathbf{y}}, \mathbf{p}_{1}\right)\right|^{2}-\int d \hat{\mathbf{y}}\left|f_{21}\left(p_{1} \hat{\mathbf{y}}, \mathbf{p}_{1}\right)\right|^{2}=0
$$

where $f_{11}$ is the elastic $e^{+}-\mathrm{H}$ amplitude and $f_{21}$ is the rearrangement Ps $-p$ amplitude. Therefore, the right hand side of (67) has the limit

$$
\frac{4 \pi}{p_{1}} \Im \mathrm{m} F_{11}^{0}\left(\mathbf{p}_{1}, \mathbf{p}_{1}\right)-\int d \hat{\mathbf{y}}\left|F_{11}^{0}\left(p_{1} \hat{\mathbf{y}}, \mathbf{p}_{1}\right)\right|^{2} \rightarrow \int d \hat{\mathbf{y}}\left|f_{21}\left(p_{1} \hat{\mathbf{y}}, \mathbf{p}_{1}\right)\right|^{2}=\sigma_{21} \cdot(70)
$$

The quantity $\sigma_{21}$ is nothing but the positronium formation cross section. At the same time the formula (68) leads to an uncertainty in the limit as $g \rightarrow 0$ when the diverging integral is multiplied by the vanishing factor $g$. Actually, the resolution of this uncertainty is made by (70) and gives the relation

$$
\sigma_{2}^{a}=\sigma_{21}+\mathcal{O}(g),
$$

which clearly shows that $\sigma_{2}^{a}$ is the cross section of the annihilation after the positronium formation.

The main result of this subsection is the representation of the annihilation cross section as the sum of two terms

$$
\sigma^{a}=\sigma_{1}^{a}+\sigma_{2}^{a},
$$

where $\sigma_{2}^{a}$ is shown to represent the cross section of the annihilation after the positronium formation, which is given by the formulae (64) or (68). Therefore $\sigma_{1}^{a}$ is the direct annihilation cross section, given by (65).

The analysis made above shows that the definitions of cross sections in terms of scattering amplitudes (64, 65) are uniform and are valid below as well as above the rearrangement threshold of the positronium formation. Moreover, by construction, the integrals (51, 54) involved in the definitions of the amplitudes $F_{11}^{0}, F_{11}^{1}$ have the finite limit as $g \rightarrow 0$. This property will be used below for perturbative calculations of the amplitudes and cross sections. At the same time the integrals in representations (62, 68) 
are divergent above the positronium formation threshold if the limit $g \rightarrow 0$ is taken. That makes these formulae not suitable for perturbative methods above the positronium formation threshold.

\subsection{Absorbing annihilation potential}

In this subsection we fix the coordinate form of the absorption potential by comparing the definition (62) for $\sigma^{a}$ with QED formula for the $2 \gamma$ singlet $e^{+} e^{-}$annihilation $甘$ [2, 3]

$$
\sigma^{a}=\pi r_{0}^{2}(c / v) Z_{\text {eff }}
$$

In this formula $r_{0}$ is the classical electron radius, $c$ is the speed of light and $v$ is the incident velocity of the positron. The effective number of electrons $Z_{\text {eff }}$ participating in annihilation is given by the integral

$$
Z_{\text {eff }}=\int d \mathbf{x}_{1} d \mathbf{y}_{1}\left|\Psi^{0+}\left(\mathbf{x}_{1}, \mathbf{y}_{1}\right)\right|^{2} \delta\left(\mathbf{x}_{2}\right) .
$$

Here $\Psi^{0+}$ is the $\Psi^{01}$ solution of the $e^{+}-\mathrm{H}$ scattering problem (26, 27, 28) when the absorption potential is not taken into account. The integral in (73) is well defined below the Ps-formation threshold. In this case (72, 73) can be considered as the first order perturbation approximation to (62), since below the rearrangement threshold $\Psi^{+} \simeq \Psi^{0+}$ is the well defined first order perturbation solution (Born) to (18). This observation was used in [4] to determine the absorption potential for $2 \gamma$ singlet $e^{+} e^{-}$annihilation as

$$
\begin{aligned}
& \mathrm{i} g W_{2}\left(\mathbf{x}_{2}\right)=\mathrm{i} g \delta\left(\mathbf{x}_{2}\right) \\
& g=-\frac{e^{2}}{a_{0}} 2 \pi \alpha^{3} .
\end{aligned}
$$

Here $a_{0}$ is the Bohr radius and $\alpha$ is the fine structure constant. This potential was used in [4] to calculate the direct annihilation cross section by solving the Lippmann-Schwinger equation for T-matrix below the Ps-formation threshold.

The formula (73) cannot be extended for calculations above the Ps-formation threshold since the integral diverges. One of ways to go over the Ps-formation threshold is the use of (62) with absorption potential (74) incorporated into the Schrödinger equation. This was done in papers [5, 7] where the overall annihilation cross section $\sigma^{a}$ was computed below as well as above the Ps-formation threshold within the hypersperical close coupling technique for the time independent three-body Schrödinger equation. Paper [8] represents the solution of the time-dependent threebody Schrödinger equation with the absorption potential (74) below as well as above the Ps-formation threshold. All these papers dealt with numerical solutions of respective equations and the delta-functional singularity of the potential (74) was treated by a certain numerical approximation. Nevertheless, the analytical status of the potential (74) is not satisfactory. The delta-functional singularity is too strong and makes the Hamiltonian not well defined. This issue was not addressed in aforementioned papers and we give a portion of the necessary analysis in this subsection.

$\ddagger$ The spin-averaging factor $1 / 4$ for singlet $2 \gamma$ annihilation is implied implicitly. 
It is well known already since the papers by Fermi [9] and then Breit [10] that the three-dimensional delta-function potential can be incorporated into the Schrödinger equation only perturbatively. One of the approaches to go beyond the perturbative treatment is the use of a zero-range potential [10, 11]. There are two common ways to introduce the zero-range potential. One is imposing boundary conditions for the wavefunction at the singularity point. The other one is introducing into the Hamiltonian an additional term, which enforces the wave-function to fulfill the boundary conditions. This term can conveniently be represented in the compact form by the quasi-potential [22, 23]. We choose the second option.

The singularity caused by the zero-range potential in the case of the electronpositron interaction is located at the same point as the Coulomb singularity $-e^{2} / x_{2}$. The latter leads to the modification of the standard zero-range potential and of the respective quasi-potential. The resulting definition for the coordinate part $W_{2}$ of the absorption potential is

$$
W_{2}(\mathbf{x})=\delta(\mathbf{x}) \frac{1-n_{2} x}{1+n_{2} x \log x} \frac{d}{d x} \frac{x}{1+n_{2} x \log x}
$$

where $n_{2}=-2 \mu_{2} e^{2}$ and $\mu_{2}$ is the $e^{+} e^{-}$reduced mass. The detailed derivation of (75) involves a substantial portion of mathematics and will be published elsewhere. Some basic theorems, which define the general properties of the zero-range potential with the Coulomb modification, can be found in [24].

It is straightforward to see that in the limit $n_{2} \rightarrow 0$ the quasi-potential (75) takes the standard form

$$
W_{2}(\mathbf{x}) \rightarrow \delta(\mathbf{x}) \frac{d}{d x} x
$$

The quasi-potential i $g W_{2}\left(\mathbf{x}_{2}\right)$ enforces the following asymptotics for the wave function as $x_{2} \rightarrow 0$

$$
\Psi\left(\mathbf{x}_{2}, \mathbf{y}_{2}\right) \propto \frac{a\left(\mathbf{y}_{2}\right)}{4 \pi}\left[\frac{1}{x_{2}}+n_{2} \log x_{2}\right]+b\left(\mathbf{y}_{2}\right)+\mathcal{O}\left(x_{2} \log x_{2}\right),
$$

where $a / b=-\mathrm{i} g 2 \mu_{2} / \hbar^{2}$. This asymptotics, as usually, determines the appropriate boundary conditions, which we do not write down here explicitly. It can be shown that the action of the quasi-potential on the function with such an asymptotics is given by the formula

$$
W_{2}\left(\mathbf{x}_{2}\right) \Psi\left(\mathbf{x}_{2}, \mathbf{y}_{2}\right)=\delta\left(\mathbf{x}_{2}\right) b\left(\mathbf{y}_{2}\right) .
$$

The latter means that the action of the quasi-potential on a function $\chi\left(\mathbf{x}_{2}, \mathbf{y}_{2}\right)$, which is regular in the point $x_{2}=0$, is equivalent to the delta-function

$$
W_{2}\left(\mathbf{x}_{2}\right) \chi\left(\mathbf{x}_{2}, \mathbf{y}_{2}\right)=\delta\left(\mathbf{x}_{2}\right) \chi\left(0, \mathbf{y}_{2}\right) .
$$

This formula shows that if a matrix element of the quasi-potential is calculated between the functions $\chi, \omega$, which are regular at $x_{2}=0$, then the quasi-potential is equivalent to the delta-function, i.e.

$$
\left\langle\chi\left|W_{2}\left(\mathbf{x}_{2}\right)\right| \omega\right\rangle=\left\langle\chi\left|\delta\left(\mathbf{x}_{2}\right)\right| \omega\right\rangle .
$$




\begin{tabular}{rrrrrr}
\hline$p_{1}$ & present work & {$[26]$} & {$[27]$} & {$[28]$} & {$[32]$} \\
\hline 0.1 & 0.1484 & 0.149 & 0.1483 & 0.152 & 0.148 \\
0.2 & 0.1879 & 0.189 & 0.1877 & 0.188 & 0.187 \\
0.3 & 0.1676 & 0.169 & 0.1677 & 0.166 & 0.167 \\
0.4 & 0.1198 & 0.121 & 0.1201 & 0.118 & 0.119 \\
0.5 & 0.0618 & 0.062 & 0.0624 & 0.061 & 0.062 \\
0.6 & 0.0032 & 0.003 & 0.0039 & 0.003 & 0.003 \\
0.7 & -0.0502 & -0.05 & -0.051 & 0.0053 & \\
\hline
\end{tabular}

Table 1. $L=0$ phase shift $\delta_{0}$ for $e^{+}-\mathrm{H}$ elastic scattering. Momenta $p_{1}$ are given in units of $a_{0}^{-1}$.

This statement justifies the use of the delta-function as the coordinate part of the absorption potential in [5, 7, 8], since the basis functions of the approaches used to compute the matrix elements of the absorption potential are smooth. Nevertheless, any basis of smooth functions cannot reproduce the singularity in (77) by a finite number of terms, what always happens along the numerical solution. Hence, such a treatment of the zero-range potential in [5, 7, 8] is approximative but, in view of the fact $a\left(\mathbf{y}_{2}\right)=-\mathrm{i} g 2 \mu_{2} b\left(\mathbf{y}_{2}\right) / \hbar^{2}$ with $|g| \ll 1$ for the $e^{+} e^{-}$annihilation, the approximation is reasonable.

\section{Calculation of annihilation cross section}

The formalism developed above was applied for calculations of the annihilation in $e^{+}-\mathrm{H}$ collision below the Ps-formation threshold and above the threshold in the Ore gap. The latter is defined as the interval of the energy between the $\operatorname{Ps}(1 s)$ and $\mathrm{H}(n=2)$ thresholds. All calculations were made on the basis of an extension of the multichannel numerical algorithm for Faddeev equations, described in details in [25], for the case of annihilation. The algorithm uses the bipolar harmonic expansion to represent the angular dependence of the wave-function components

$$
\begin{aligned}
& \Psi_{i}^{+}\left(\mathbf{x}_{i}, \mathbf{y}_{i}\right)=\sum_{L l_{1} l_{2}} \frac{\psi_{l_{1} l_{2}}^{L}\left(x_{i}, y_{i}\right)}{x_{i} y_{i}} \mathcal{Y}_{l_{1} l_{2}}^{L M}\left(\hat{\mathbf{x}}_{i}, \hat{\mathbf{y}}_{i}\right), \\
& \mathcal{Y}_{l_{1} l_{2}}^{L M}\left(\hat{\mathbf{x}}_{i}, \hat{\mathbf{y}}_{i}\right)=\left[Y_{l_{1}}^{m_{1}}\left(\hat{\mathbf{x}}_{i}\right) \otimes Y_{l_{2}}^{m_{2}}\left(\hat{\mathbf{y}}_{i}\right)\right]_{L M} .
\end{aligned}
$$

This expansion reduces the MFE to a set of coupled equations for radial components $\psi_{l_{1}, l_{2}}^{L}\left(x_{i}, y_{i}\right)$ which are then approximated by the quintic-spline expansion and solved by the orthogonal collocation procedure. The maximum values of $l_{1}, l_{2}$ used in (81) range from 12 to 15.

In order to test the numerical approach, the genuine Coulomb problem for $e^{+}-\mathrm{H}$ scattering was solved on the platform of MFE (32, 33) with $g=0$. The results of calculations for s-wave $e^{+}-\mathrm{H}$ phase shift $\delta_{0}$ are given in Table 1 together with data of other authors. As one can see, the agreement of our calculations with previous results 


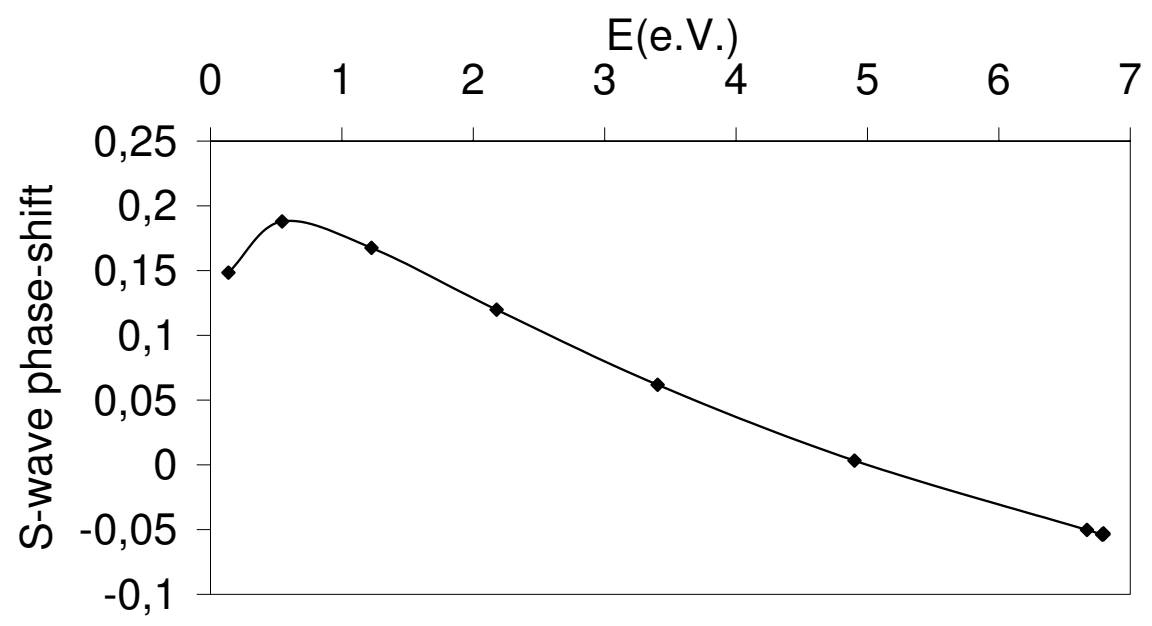

Figure 1. $L=0$ phase-shift for $e^{+}-\mathrm{H}$ elastic scattering.

is quite good. Figure 1 provides an alternative representation of $\delta_{0}$ as the function of the energy to demonstrate the regular character of the calculated phase shift $\delta_{0}$. The calculations of the direct annihilation cross section $\sigma_{1}^{a}$ were performed with the help of the representation (65)). The amplitudes $F_{11}^{0}$ and $F_{11}^{1}$ were computed from the solutions of the respective Faddeev equations. The effective number $Z_{\text {eff }}^{1}$ for the direct annihilation cross section was obtained using the standard expression (72)

$$
\sigma_{1}^{a}=\pi r_{0}^{2}(c / v) Z_{\text {eff }}^{1}
$$

As it was mentioned in subsection 3.1, in the calculations of the amplitudes $F_{11}^{0}$ and $F_{11}^{1}$ we have systematically approximated the Faddeev components, involved in the matrixelements for the amplitudes, by the solutions $\Psi_{i}^{00 \pm}$ of the MFE with $g=0$. This statement can easily be justified by the iterative solution of the IMFE (36, 37) for $|g| \ll 1$. The iterative solutions is well defined thanks to the fact that the matrix kernel of the Faddeev equation is proven to be compact. This is another advantage of the Faddeev three-body equations. As the result, the expression for the absorption amplitude $F_{11}^{1}$ from (54) can be simplified as

$$
F_{11}^{1}\left(\mathbf{p}_{1}^{\prime}, \mathbf{p}\right)=\frac{-\nu_{1}}{2 \pi \hbar^{2}}\left\langle\left[\Psi_{1}^{00-}\left(\mathbf{p}_{1}^{\prime}\right)+\Psi_{2}^{00-}\left(\mathbf{p}_{1}^{\prime}\right)\right]\left|W_{2}\right| \Psi_{1}^{00+}\left(\mathbf{p}_{1}\right)\right\rangle,
$$

which was actually used for calculations of this paper.

Since a number of data from other authors for direct annihilation cross section below the Ps-formation threshold is available, In Table 2 we display our calculated phase-shift $\delta_{0}$ and $Z_{\text {eff }}^{1}$ together with results of other authors obtained with the standard formula (72) for one of the typical value of the relative momentum $p_{1}=0.4\left[1 / a_{0}\right]$. The agreement is very good for calculations made by quite different approaches. In Table 3 we collect the results of existing calculations of $Z_{\text {eff }}$ below the Ps-formation threshold to compare with our results. One cannot expect the complete agreement since our definition of $\sigma_{1}^{a}$ concerns the direct process only, whereas the standard definition below the Ps-formation 


\begin{tabular}{rrr}
\hline Ref. & $\delta_{0}$ & $Z_{\text {eff }}$ \\
\hline present work & 0.11983 & 3.3293 \\
{$[29$} & 0.1198 & 3.232 \\
27 & 0.1201 & 3.327 \\
30 & 0.1198 & 3.407 \\
31 & 0.1191 & 3.332 \\
\hline
\end{tabular}

Table 2. $L=0$ phase shift $\delta_{0}$ for $e^{+}-\mathrm{H}$ elastic scattering and effective number $Z_{\text {eff }}$ for the relative momentum $p_{1}=0.4\left[a_{0}^{-1}\right]$.

\begin{tabular}{rrrrr}
\hline$p_{1}$ & present paper & {$[26]$} & {$[32]$} & {$[33]$} \\
\hline 0.1 & 7.2570 & 7.55 & 7.5 & 7.363 \\
0.2 & 5.1627 & 5.74 & 5.7 & 5.538 \\
0.3 & 4.1061 & 4.36 & 4.3 & 4.184 \\
0.4 & 3.3293 & 3.4 & 3.3 & 3.327 \\
0.5 & 2.8118 & 2.74 & 2.7 & 2.73 \\
0.6 & 2.4625 & 2.29 & 2.3 & 2.279 \\
0.7 & 2.2529 & 2.02 & & 1.950 \\
\hline
\end{tabular}

Table 3. $L=0$ effective number $Z_{\text {eff }}$ for $e^{+}-\mathrm{H}$ annihilation. The relative momenta $p_{1}$ are given in units of $a_{0}^{-1}$.

threshold deals with the overall annihilation cross section $\sigma^{a}$. Nevertheless, Table 3 shows that the difference is not so dramatic.

The extensive calculations of the direct annihilation cross section $\sigma_{1}^{a}$ were made in the interval of the energy between $\mathrm{H}(n=1)$ and $\mathrm{H}(n=2)$ thresholds on the basis of the formula (65). Figure 2 shows the s-wave effective number $Z_{\text {eff }}^{1}$ derived from $\sigma_{1}^{a}$, with the formula (82), and the results of calculations from [8]. Although, quite different methods (time-independent Faddeev equations and time-dependent wave-packet approach to the Schrödinger equation) of calculations for the direct annihilation cross sections have been used, the agreement between data is fairly good. In fact, the definition of the direct cross section $\sigma_{1}^{a}$ in our formalism as the remainder of the overall annihilation cross section $\sigma^{a}$ after subtraction of the annihilation cross section after the Ps-formation $\sigma_{2}^{a}$

$$
\sigma_{1}^{a}=\sigma^{a}-\sigma_{2}^{a},
$$

(see equation (63) ) is quite equivalent to the time-dependent definition by formula (7) of ref. [8]. Nevertheless, as the data of Tables 4, 5] shows, the exact use of the formula (84) would be very unpractical. The formation cross section $\sigma_{21}$, which is the leading term of $\sigma_{2}^{a}$ due to (71), rapidly increases above the threshold and is several order of magnitude bigger than $\sigma_{1}^{a}$. The analytic separation of the overall annihilation cross section into the formation and direct parts made in Subsection 3.1 and given by formulae (63, 65) in terms of amplitudes $F_{11}^{0}$ and $F_{11}^{1}$ is therefore of the great practical importance. 


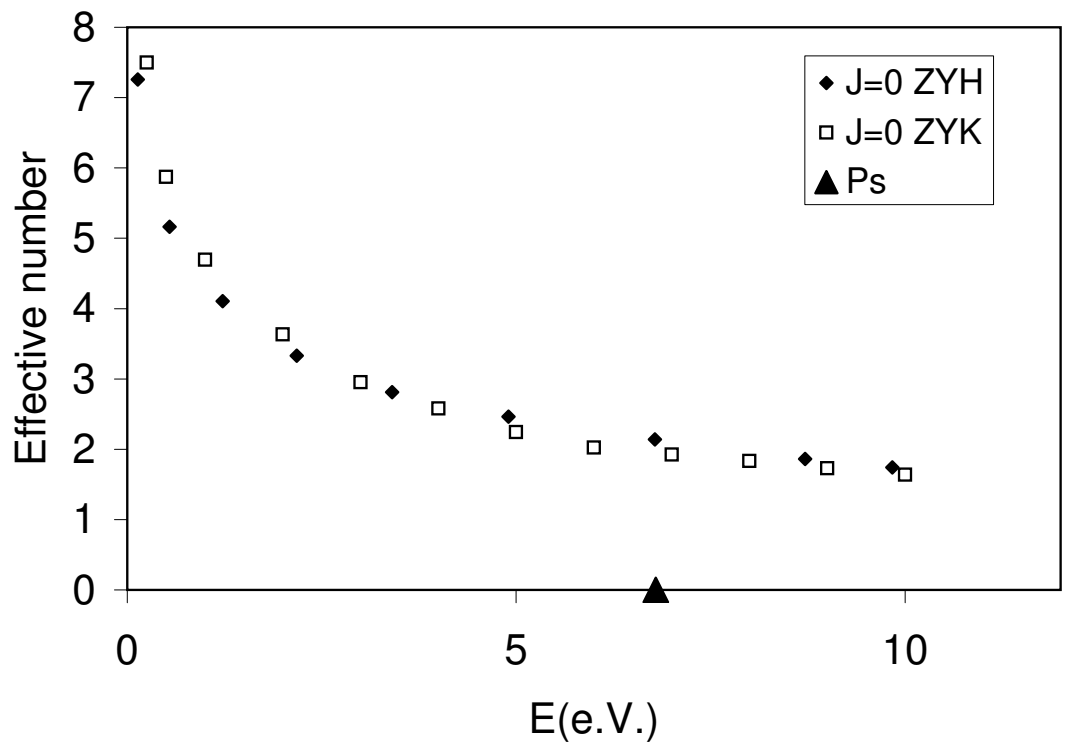

Figure 2. The effective number $Z_{\mathrm{eff}}^{1}$ for the $L=0$ direct annihilation. The diamonds are the calculations of the present paper, the open squares are the data from [8], the triangle marks the positronium formation threshold.

In the following Tables 4 and 5 we present the results of our calculations for the direct annihilation cross section together with the cross section of the positronium formation above the Ps-formation threshold. They are given for slightly different values of momenta $p_{1}$ for $L=0$ and $L=1$ in order to emphasize the most characteristic behavior of cross sections near the respective Feshbach resonances. Besides the expected difference in several order of magnitude between direct annihilation cross section $\sigma_{1}^{a}$ and the positronium formation cross section $\sigma_{21}$, the strong correlation between these cross sections in the region of the sharp increase of $\sigma_{1}^{a}$ across $0.86124\left[a_{0}^{-1}\right]$ for $L=0$ and $0.86318\left[a_{0}^{-1}\right]$ for $L=1$ is clearly seen. The graphical representation of that sharp increase of cross sections is given on Figures 3 and 4 which display s- and p-wave $Z_{\text {eff }}^{1}$. This resonant feature was also observed in the spatial behavior of the solution to the Faddeev equation. The first component $\Psi_{1}^{+}$, which is related to the $e^{+}-\mathrm{H}$ channel, always exhibits the characteristic resonant bump near resonant energy. These spatial resonant structures can be extensive depending on the lifetime of the resonances. In [13, reasonable estimation of the energy width of a number of Feshbach resonances were made using the uncertainty principle applied to the graphical representation of the wave function components.

\section{Conclusion}

In present paper we have developed the multichannel time-independent formalism, which is capable to describe the scattering and annihilation processes in the positron-hydrogen collision above the rearrangement threshold. The expression derived in the paper for the 


\begin{tabular}{rrrr}
\hline$p_{1}$ & $Z_{\text {eff }}^{1}$ & $\sigma_{1}^{a}$ & $\sigma_{21}$ \\
\hline 0.70654 & 2.3289 & $1.28[-6]$ & $9.05[-4]$ \\
0.71 & 2.1715 & $1.19[-6]$ & $4.14[-3]$ \\
0.8 & 1.8640 & $9.05[-7]$ & $5.03[-3]$ \\
0.85 & 1.7404 & $7.95[-7]$ & $5.83[-3]$ \\
0.861 & 1.4840 & $6.69[-7]$ & 0.01087 \\
0.8611 & 1.9678 & $8.88[-7]$ & 0.01682 \\
0.86118 & 2.2200 & $1.00[-6]$ & 0.02694 \\
0.86119 & 2.3022 & $1.04[-6]$ & 0.02943 \\
0.8612 & 2.4175 & $1.09[-6]$ & 0.03247 \\
0.86121 & 2.5832 & $1.16[-6]$ & 0.03622 \\
0.86122 & 2.7817 & $1.25[-6]$ & 0.04087 \\
0.86124 & 3.1700 & $1.43[-6]$ & 0.05380 \\
0.86126 & 0.9735 & $4.39[-7]$ & 0.07030 \\
0.86128 & 0.9742 & $4.39[-7]$ & 0.07450 \\
0.86132 & 1.4915 & $6.73[-7]$ & 0.02089 \\
0.8614 & 1.6459 & $7.42[-7]$ & 0.00013 \\
0.8615 & 1.7034 & $7.68[-7]$ & 0.00083 \\
0.8618 & 1.7674 & $7.97[-7]$ & 0.00326 \\
\hline
\end{tabular}

Table 4. $L=0$ effective number $Z_{\text {eff }}^{1}$, the direct annihilation cross section $\sigma_{1}^{a}$, and the positronium formation cross section $\sigma_{21}$. The cross sections are given in units of $\pi a_{0}^{2}$ and momenta in units of $a_{0}^{-1}$. The abbreviation [-n] is used for $10^{-n}$.

\begin{tabular}{rrrr}
\hline$p_{1}$ & $Z_{\text {eff }}^{1}$ & $\sigma_{1}^{a}$ & $\sigma_{21}$ \\
\hline 0.8 & 0.5404 & $2.62[-7]$ & 0.485 \\
0.85 & 0.6933 & $3.17[-7]$ & 0.566 \\
0.8631 & 0.4775 & $2.15[-7]$ & 0.749 \\
0.86313 & 0.4695 & $2.11[-7]$ & 0.848 \\
0.86315 & 0.4675 & $2.10[-7]$ & 1.022 \\
0.86317 & 0.5914 & $2.66[-7]$ & 1.772 \\
0.86318 & 4.8319 & $2.17[-6]$ & 3.680 \\
0.863185 & 14.6255 & $6.58[-6]$ & 1.770 \\
0.86319 & 2.1967 & $9.88[-7]$ & 0.129 \\
0.8632 & 0.9012 & $4.06[-7]$ & 0.068 \\
0.86325 & 0.5629 & $2.53[-7]$ & 0.407 \\
\hline
\end{tabular}

Table 5. $L=1$ effective number $Z_{\text {eff }}^{1}$, the direct annihilation cross section $\sigma_{1}^{a}$, and the positronium formation cross section $\sigma_{21}$. The cross sections are given in units of $\pi a_{0}^{2}$ and momenta in units of $a_{0}^{-1}$. The abbreviation [-n] is used for $10^{-n}$. 


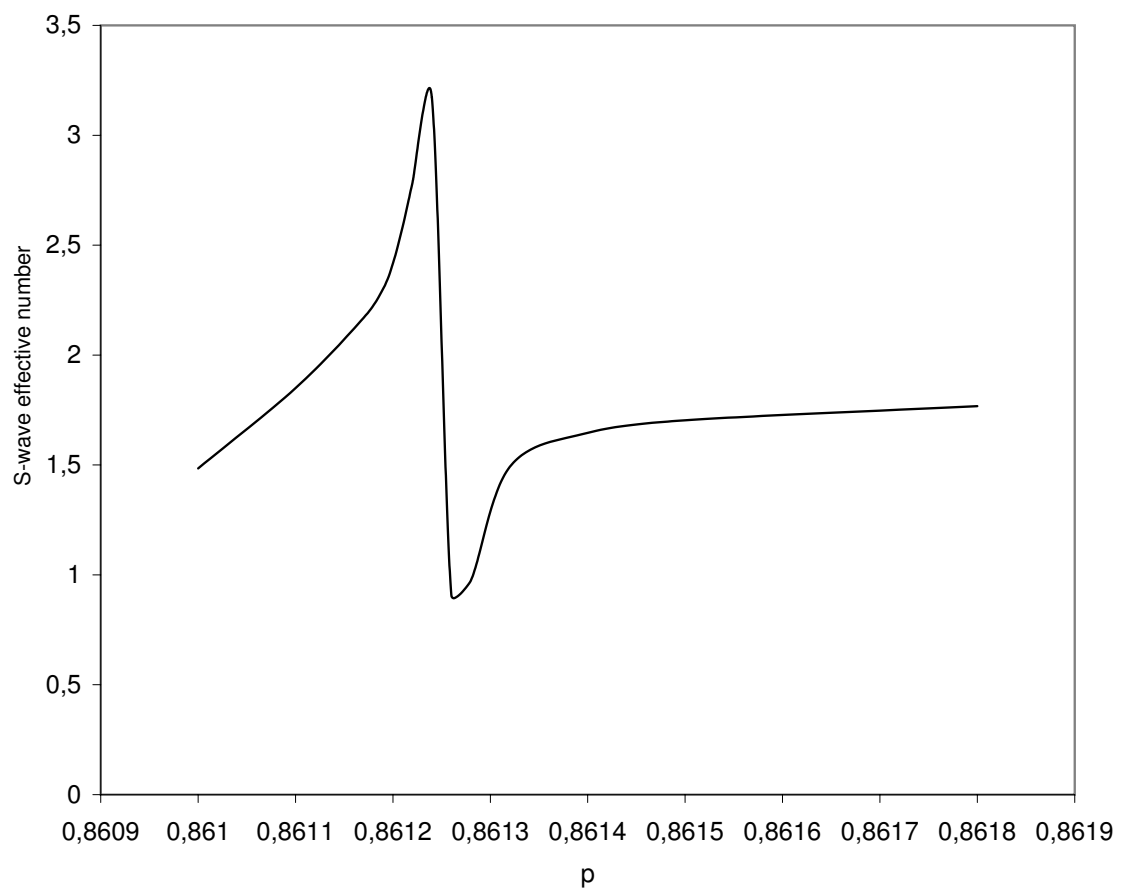

Figure 3. $L=0$ effective number $Z_{\text {eff }}^{1}$ in the resonant region. The momenta $p$ are given in $a_{0}^{-1}$ units.

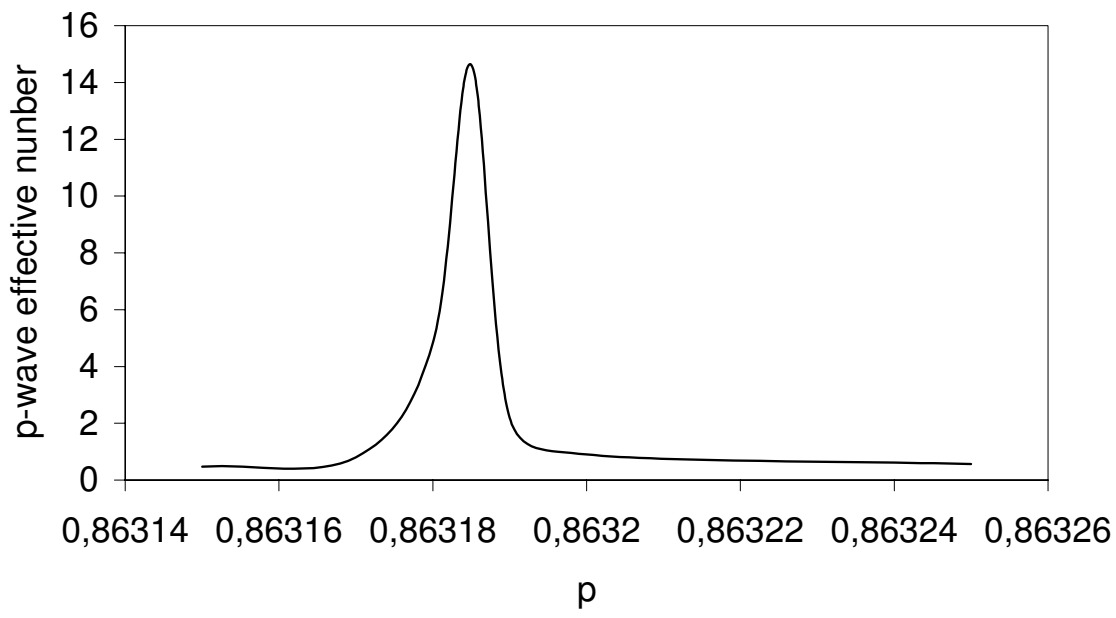

Figure 4. $L=1$ effective number $Z_{\text {eff }}^{1}$ in the resonant region. The momenta $p$ are given in $a_{0}^{-1}$ units. 
direct annihilation cross section in terms of amplitudes is proven to extend the standard formula to the energy region above the positronium formation threshold. Below the threshold our cross section and the cross section calculated from the standard theory are in good agreement provided the close vicinity of the positronium-formation threshold is not considered where the standard cross section becomes infinite. The direct annihilation cross section defined by the formula (65) does not exhibit any singular behavior at the Ps-formation threshold. It is in good agreement with the nonsingular direct annihilation cross section computed from the time-dependent solution of the three-body Schrödinger equation for $e^{+}-\mathrm{H}$ system [8].

The formalism of this paper can readily be extended beyond the Ore gap. In this case the multichannel optical theorem, which generalizes (61), plays the key role in the determination of cross sections. Preliminary calculations indicated the much larger enhancement of the direct annihilation cross section near the Feshbach resonances in the eight open channel region above the $\operatorname{Ps}(n=2)$ threshold. Since there are numerous resonances beyond the Ore gap, they evidently made a significant contribution to the overall annihilation peak around $12 \mathrm{e.V}$. displayed in Figure 2 of [8]. We hope that our individual annihilation resonance structure will provide a new experimental tool to study sharp resonances. Although at the present time, it is not possible to conduct such an experiment for $e^{+}-\mathrm{H}$ system, experiments for positron scattering on large molecules have been done for many systems [34].

Our approach, in perspective, opens the way to considering more complicated systems with more than three particles, as for example $e^{+} e^{+} e^{-} e^{-}$. Suitable formalism for multichannel scattering [35], which is the generalization of the Faddeev equations for the four-particle systems, will be helpful as an important theoretical step towards the experimental verification and utilization of the rich positron annihilation physics.

\section{Acknowledgments}

The authors appreciate the support of the NSF grant Phy-0243740, INTAS grant No. 0351-4000 and the generous supercomputer time awards from grants MCA96T011 and TGMCA96T011 under the NSF partnership for Advanced Computational Infrastructure, Distributed Terascale Facility (DTF) to the Extensible Terascale Facility. In particular we are thankful to PSC and SDSC. The authors would like to thank Dr. Y. Kino for providing us with numerical data corresponding to the Figure 2 of ref. [8], which made the comparison of our results much feasible. We are thankful to Prof. K.A. Makarov for fruitful discussion on zero-range potentials.

\section{References}

[1] Rich A 1981 Rev. Mod. Phys. 53127

[2] Fraser P A 1968 Adv. At. Mol. Phys. 463

[3] Charlton M, Humberstone J W 2001 Positron Physics (Cambridge, UK: Cambridge University Press) 
[4] Ivanov I A, Mitroy J 2000 J. Phys. B: At. Mol. Opt. Phys. 33 L831

[5] Igarashi A, Kimura M, Shimamura I 2002 Phys. Rev. Lett. 89123201

[6] Gribakin G F, Ludlow J 2002 Phys. Rev. Lett. 88163202

[7] Igarashi A, Kimura M, Shimamura I, Toshima N 2003 Phys. Rev. A 68042716

[8] Yamanaka N, Kino Y, Takano Y, Kudo H 2003 Phys. Rev. A 67052712

[9] Fermi E 1936 Ricerca Sci. 713

[10] Breit G 1947 Phys. Rev. 71215

[11] Demkov Yu N, Ostrovskii V N 1988 Zero-Range Potentials and their Applications in Atomic Physics (New York: Plenum)

[12] Van Reeth P, Laricchia G, Humberston J W 2005 Phys. Scripta 71 C9-13

[13] Hu C-Y, Yakovlev S L, Papp Z 2006 Nucl. Instr. Meth. B 24725

[14] Messia A 1958 Quantum Mechanics (New-York: J Wiley and Sons, Inc. )

[15] Foldy L L, Tobocman W 1957 Phys. Rev. 1051099

[16] Newton R G 1982 Scattering Theory of Waves and Particles (New-York: Springer-Verlag NewYork Inc.)

[17] Schmid E W, Ziegelman H 1974 The quantum mechanical three-body problem (Braunschweig: Vieweg)

[18] Faddeev L D 1961 Sov. Phys. JETP 121014

Faddeev L D, Merkuriev S P 1993 Quantum Scattering Theory for Several Particle Systems (Dordrech: Kluver)

[19] Merkuriev S P Ann. Phys. (NY) 130395

[20] Yakovlev S L 1996 Theor. Math. Phys. 107835

Yakovlev S L 1999 Few Body Systems Supplement 1085

Evans J W 1981 J. Math. Phys. 221672

Evans J W, Hoffman D K 1981 J. Math. Phys. 222858

[21] Messia A 1958 Quantum Mechanics (New-York: J Wiley and Sons, Inc.), see CH. XIX, §2 Eq. XIX.14

[22] Blatt J M, Weisskopf V F 1952 Theoretical Nuclear Physics (New-York: J Willey and Sons, Inc.)

[23] Huang K, Yang C N 1957 Phys. Rev. 105767

[24] Albeverio S, Gesztesy F, Høegh-Krohn R, Holden H 2005 Solvable Models in Quantum Mechanics (Providence, Rhode Island: AMS Chelsea Publishing)

[25] Hu C-Y 1999 J. Phys. B: At. Mol. Opt. Phys. 32, 3077

[26] Kvitsinsky A A, Wu A, Hu C-Y 1995 J. Phys. B: At. Mol. Opt. Phys. 28275

[27] Bhatia A K, Temkin A, Drachman R J, Eiserike H 1971 Phys. Rev. A 31328

[28] Levin F S, Shetzer J 1988 Phys. Rev. Lett. 611089

[29] Bromley M W J, Mitroy J 2003 Phys. Rev. A 67062709

[30] Gribakin G F, Ludlow J 2003 Phys. Rev. A 70032720 Van Reeth P, Humberston J W 1997 J. Phys. B: At. Mol. Opt. Phys. 302477

Van Reeth P, Humberston J W 1998 J. Phys. B: At. Mol. Opt. Phys. 31 L231

[31] Mitroy J, Ratnavelu K 1995 J. Phys. B: At. Mol. Opt. Phys. 28287

Ryzhikh G G, Mitroy J 2000 J. Phys. B: At. Mol. Opt. Phys. 332229

[32] Humberstone J W, Wallace J B G 1972 J. Phys. B: At. Mol. Phys. 51138

[33] Bhatia A K, Drachman R J, Temkin A 1974 Phys. Rev. A 9223

[34] Barnes L D, Gilbert S J, Surko C M 2003 Phys. Rev. A 67, 032706 Surko C M, Gribakin G F, Buckman S J 2005 J. Phys. B: At. Mol. Opt. Phys. 38 R1-R70

Barnes L D, Young J A, Surko C M 2006 Phys. Rev. A 74012706

[35] Merkuriev S P, Yakovlev S L 1982 Doklady AN USSR 262 No. 3, 591

Merkuriev S P, Yakovlev S L, Gignoux C 1984 Nucl. Phys. A431 125 